\title{
Enabling Versus Controlling - Online Appendix
}

\section{Citation}

Hagiu, Andrei, and Julian Wright. "Enabling Versus Controlling-Online Appendix." Harvard Business School Working Paper, No. 16-004, July 2015. (Revised October 2015.)

\section{Permanent link}

http://nrs.harvard.edu/urn-3:HUL.InstRepos:17527691

\section{Terms of Use}

This article was downloaded from Harvard University's DASH repository, and is made available under the terms and conditions applicable to Open Access Policy Articles, as set forth at http:// nrs.harvard.edu/urn-3:HUL.InstRepos:dash.current.terms-of-use\#OAP

\section{Share Your Story}

The Harvard community has made this article openly available.

Please share how this access benefits you. Submit a story.

Accessibility 
H A R VAR D

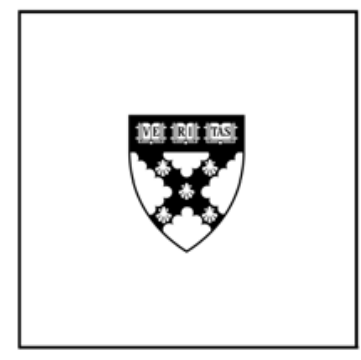

\title{
Enabling Versus Controlling - Online Appendix
}

\author{
Andrei Hagiu \\ Julian Wright
}

\section{Working Paper}

16-004

October 28, 2015 


\title{
Enabling versus controlling - Online appendix
}

\author{
Andrei Hagiu* and Julian Wright ${ }^{\dagger}$
}

October 28, 2015

This online appendix contains the derivation of the closed form solutions for the examples used in the main paper. It establishes the result stated in Section 4.3 of the main paper, that Proposition 4 continues to hold even if the price is endogenous and there are production costs. It provides the proofs of some results stated in the extensions section of the paper. It also details the numerical analysis performed to check the robustness of the first part of Corollary 1 to the introduction of private benefits and spillovers.

\section{Linear example}

We directly conduct the calculations for the linear example with $N>1$ agents and spillovers, i.e. where the revenue generated by agent $i$ is

$$
R_{i}=\theta a_{i}+x\left(\bar{a}_{-i}-a_{i}\right)+\gamma e_{i}+\delta I
$$

and fixed costs are

$$
c_{i}^{a}(a)=\frac{1}{2} a^{2}, \quad c_{i}^{e}(e)=\frac{1}{2} e^{2} \quad \text { and } \quad c^{I}(I)=\frac{1}{2} I^{2} .
$$

The results for the case $N=1$ are then simply derived by setting $x=0$ and $N=1$.

Consider first the $E$-mode. The payoff to agent $i$ from working for the firm is

$$
(1-t) R_{i}-\frac{1}{2} e_{i}^{2}-T=(1-t)\left(\theta a_{i}+x\left(\bar{a}_{-i}-a_{i}\right)+\gamma e_{i}+\delta I\right)-\frac{1}{2} e_{i}^{2}-T
$$

which implies that the level of effort chosen by each agent in the second stage is

$$
e^{E}(t)=\gamma(1-t)
$$

*Harvard Business School, Boston, MA 02163, E-mail: ahagiu@hbs.edu

${ }^{\dagger}$ Department of Economics, National University of Singapore, Singapore 117570, E-mail: jwright@nus.edu.sg 
In $E$-mode, the firm sets $a_{1}, \ldots, a_{N}$ and $I$ to maximize its second stage revenues (wages are paid in the first stage):

$$
\sum_{i=1}^{N}\left(t\left(\theta a_{i}+x\left(\bar{a}_{-i}-a_{i}\right)+\gamma e_{i}+\delta I\right)-\frac{1}{2} a_{i}^{2}\right)-\frac{1}{2} I^{2},
$$

implying the firm's optimal choices are

$$
\begin{aligned}
& a^{E}(t)=\theta t \\
& I^{E}(t)=N \delta t .
\end{aligned}
$$

The fixed fee $T$ is set to render each agent indifferent between working for the firm and her outside option, so the expression of $E$-mode profits as a function of $t$ is

$$
\frac{N}{2}\left(\left(\theta^{2}+N \delta^{2}\right) t(2-t)+\gamma^{2}\left(1-t^{2}\right)\right)
$$

Maximizing (2) with respect to $t$ implies the optimal variable fee in $E$-mode is

$$
t^{E *}=\frac{\theta^{2}+N \delta^{2}}{\theta^{2}+\gamma^{2}+N \delta^{2}}
$$

which is positive but smaller than 1 . With this optimal variable fee, the resulting profits in $E$-mode are

$$
\Pi^{E *}=\frac{N}{2}\left(\theta^{2}+N \delta^{2}+\frac{\gamma^{4}}{\theta^{2}+\gamma^{2}+N \delta^{2}}\right) .
$$

Consider next the $P$-mode. The payoff to an individual agent joining the platform is

$$
(1-t)\left(\theta a_{i}+x\left(\bar{a}_{-i}-a_{i}\right)+\gamma e_{i}+\delta I\right)-\frac{1}{2} a_{i}^{2}-\frac{1}{2} e_{i}^{2}-T .
$$

Individual agents maximize their second stage payoff by choosing

$$
\begin{gathered}
e^{P}(t)=\gamma(1-t) \\
a^{P}(t)=(\theta-x)(1-t) .
\end{gathered}
$$

The firm's second stage profits in $P$-mode are

$$
\sum_{i=1}^{N} t\left(\theta a_{i}+x\left(\bar{a}_{-i}-a_{i}\right)+\gamma e_{i}+\delta I\right)-\frac{1}{2} I^{2}
$$


which the firm maximizes over $I$, leading to

$$
I^{P}(t)=N \delta t
$$

Stepping back to the first stage, the firm sets $T$ to equalize the agents' net payoff to their outside option. Total firm profit in $P$-mode as a function of $t$ is then

$$
\frac{N}{2}\left((\theta-x)(1-t)(\theta+x+(\theta-x) t)+\gamma^{2}\left(1-t^{2}\right)+N \delta^{2} t(2-t)\right) .
$$

The optimal variable fee is

$$
t^{P *}=\frac{N \delta^{2}-x(\theta-x)}{(\theta-x)^{2}+\gamma^{2}+N \delta^{2}} .
$$

Resulting profits in $P$-mode are

$$
\Pi^{P *}=\frac{N}{2}\left(\theta^{2}-x^{2}+\gamma^{2}+\frac{\left(N \delta^{2}-x(\theta-x)\right)^{2}}{(\theta-x)^{2}+\gamma^{2}+N \delta^{2}}\right) .
$$

Comparing (3) with (5), the $P$-mode is preferred if and only if

$$
\gamma^{2}+\frac{\left(N \delta^{2}-x(\theta-x)\right)^{2}}{(\theta-x)^{2}+\gamma^{2}+N \delta^{2}}>N \delta^{2}+x^{2}+\frac{\gamma^{4}}{\theta^{2}+\gamma^{2}+N \delta^{2}}
$$

If there are no spillovers, i.e. $x=0$, then this condition simplifies to

$$
\gamma^{2}>N \delta^{2}
$$

For $x \neq 0$, the condition can be re-written

$$
\left(\frac{\gamma^{2} x}{\theta}+\theta^{2}+N \delta^{2}\right)^{2} \leq \theta^{2}\left(\theta^{2}+\gamma^{2}+N \delta^{2}\right)+\gamma^{4}
$$

or

$\frac{\theta}{\gamma^{2}}\left(-\left(\theta^{2}+N \delta^{2}\right)-\sqrt{\theta^{2}\left(\theta^{2}+\gamma^{2}+N \delta^{2}\right)+\gamma^{4}}\right) \leq x \leq \frac{\theta}{\gamma^{2}}\left(-\left(\theta^{2}+N \delta^{2}\right)+\sqrt{\theta^{2}\left(\theta^{2}+\gamma^{2}+N \delta^{2}\right)+\gamma^{4}}\right)$.

Finally, let us determine the effects of $\gamma$ and $\delta$ on the tradeoff between the two modes. To do so, 
we apply the envelope theorem to expressions (2) and (4) and obtain

$$
\begin{aligned}
& \frac{d \Pi^{E *}}{d\left(\gamma^{2}\right)}=\frac{N}{2}\left(1-\left(t^{E *}\right)^{2}\right) \text { and } \frac{d \Pi^{E *}}{d\left(N \delta^{2}\right)}=\frac{N}{2} t^{E *}\left(2-t^{E *}\right) \\
& \frac{d \Pi^{P *}}{d\left(\gamma^{2}\right)}=\frac{N}{2}\left(1-\left(t^{P *}\right)^{2}\right) \text { and } \frac{d \Pi^{P *}}{d\left(N \delta^{2}\right)}=\frac{N}{2} t^{P *}\left(2-t^{P *}\right)
\end{aligned}
$$

Since $0<t^{E *}, t^{P *}<1$ and $t(2-t)$ is increasing in $t$ for $t \in[0,1]$, we conclude that

$$
\begin{aligned}
& \frac{d\left(\Pi^{P *}-\Pi^{E *}\right)}{d\left(\gamma^{2}\right)}>0 \text { if and only if } t^{E *}>t^{P *} \\
& \frac{d\left(\Pi^{E *}-\Pi^{P *}\right)}{d\left(N \delta^{2}\right)}>0 \text { if and only if } t^{E *}>t^{P *} .
\end{aligned}
$$

\section{Linear example: endogenous price and production costs}

We now extend the linear example from the previous section with $x=0$ and $N=1$, by allowing the firm to also set a price in the contracting stage, along with the fees $(t, T)$, and by also adding a production cost. We will establish the result stated at the end of Section 4.3 in the main paper, i.e. that Proposition 4 continues to hold in this case.

The revenue generated by the agent is now

$$
R(p, a, e, I)=(p-d)\left(D_{0}+\theta a+\gamma e+\delta I-p\right),
$$

where $d \geq 0$ is a constant marginal production cost, $p$ is the price chosen by the firm and $D_{0}$ is some baseline level of demand. Fixed costs are still quadratic

$$
c^{a}(a)=\frac{1}{2} a^{2}, \quad c^{e}(e)=\frac{1}{2} e^{2} \quad \text { and } \quad c^{I}(I)=\frac{1}{2} I^{2}
$$

First, we show that whether the production cost is incurred by the firm or the agent does not affect profits in either mode. In $E$-mode, if the firm incurs the production cost, then the maximization 
problem is

$$
\begin{aligned}
\widetilde{\Pi}^{E *}= & \max _{p, t, a, e, I}\left\{(p-d)\left(D_{0}+\theta a+\gamma e+\delta I-p\right)-\frac{1}{2} a^{2}-\frac{1}{2} e^{2}-\frac{1}{2} I^{2}\right\} \\
\text { s.t. } & \left\{\begin{array}{l}
(t p-d) \theta=a \\
(1-t) \gamma p=e \\
(t p-d) \delta=I .
\end{array}\right.
\end{aligned}
$$

If instead the agent incurs the production cost, then the maximization problem is

$$
\begin{aligned}
\widetilde{\Pi}^{E *}= & \max _{p \widetilde{t}, a, e, I}\left\{(p-d)\left(D_{0}+\theta a+\gamma e+\delta I-p\right)-\frac{1}{2} a^{2}-\frac{1}{2} e^{2}-\frac{1}{2} I^{2}\right\} \\
\text { s.t. } & \left\{\begin{array}{c}
\widetilde{t} p \theta=a \\
((1-\widetilde{t}) p-d) \gamma=e \\
\widetilde{t} p \delta=I .
\end{array}\right.
\end{aligned}
$$

By making the change of variables $\widetilde{t} \equiv t-\frac{d}{p}$, the second maximization problem becomes the same as the first.

Similarly, in $P$-mode, if the firm incurs the production cost, then the maximization problem is

$$
\begin{aligned}
\widetilde{\Pi}^{P *}= & \max _{p, t, a, e, I}\left\{(p-d)\left(D_{0}+\theta a+\gamma e+\delta I-p\right)-\frac{1}{2} a^{2}-\frac{1}{2} e^{2}-\frac{1}{2} I^{2}\right\} \\
\text { s.t. } & \left\{\begin{array}{l}
(1-t) p \theta=a \\
(1-t) p \gamma=e \\
(t p-d) \delta=I .
\end{array}\right.
\end{aligned}
$$

If instead the agent incurs the production cost, then the maximization problem is

$$
\begin{aligned}
\widetilde{\Pi}^{P *}= & \max _{p \widetilde{t}, a, e, I}\left\{(p-d)\left(D_{0}+\theta a+\gamma e+\delta I-p\right)-\frac{1}{2} a^{2}-\frac{1}{2} e^{2}-\frac{1}{2} I^{2}\right\} \\
\text { s.t. } & \left\{\begin{array}{c}
((1-\widetilde{t}) p-d) \theta=a \\
((1-\widetilde{t}) p-d) \gamma=e \\
\widetilde{t} p \delta=I .
\end{array}\right.
\end{aligned}
$$

Again, by making the change of variables $\widetilde{t}=t-\frac{d}{p}$, the second maximization problem becomes the 
same as the first. Thus, in our setting it is irrelevant which party actually incurs the production cost.

Solving the program above in $E$-mode, we obtain

$$
\widetilde{\Pi}^{E *}=\max _{p, t}\left\{(p-d)\left(D_{0}-p\right)+\frac{\theta^{2}+\delta^{2}}{2}(t p-d)((2-t) p-d)+\frac{\gamma^{2}}{2} p(1-t)(p(1+t)-2 d)\right\} .
$$

Holding $p$ fixed and optimizing over $t$, we obtain

$$
t^{E *}(p)=\frac{\left(\theta^{2}+\delta^{2}\right) p+\gamma^{2} d}{\left(\theta^{2}+\delta^{2}+\gamma^{2}\right) p}
$$

Substituting this back into $\widetilde{\Pi}^{E *}$, the program becomes

$$
\widetilde{\Pi}^{E *}=\max _{p}\left\{(p-d)\left(D_{0}-p\right)+(p-d)^{2} \Pi^{E *}\right\}
$$

where $\Pi^{E *}$ is given by (3) with $x=0$ and $N=1$. Similarly, solving the program above in $P$-mode, we have

$$
\widetilde{\Pi}^{P *}=\max _{p, t}\left\{(p-d)\left(D_{0}-p\right)+\frac{\delta^{2}}{2}(t p-d)((2-t) p-d)+\frac{\theta^{2}+\gamma^{2}}{2} p(1-t)(p(1+t)-2 d)\right\} .
$$

Holding $p$ fixed and optimizing over $t$, we obtain

$$
t^{P *}(p)=\frac{\delta^{2} p+\left(\theta^{2}+\gamma^{2}\right) d}{\left(\theta^{2}+\delta^{2}+\gamma^{2}\right) p}
$$

Substituting this back into $\widetilde{\Pi}^{P *}$, the program becomes

$$
\widetilde{\Pi}^{P *}=\max _{p}\left\{(p-d)\left(D_{0}-p\right)+(p-d)^{2} \Pi^{P *}\right\}
$$

where $\Pi^{P *}$ is given by (5) with $x=0$ and $N=1$.

Comparing the last expressions of $\widetilde{\Pi}^{P *}$ and $\widetilde{\Pi}^{E *}$, we can conclude that

$$
\widetilde{\Pi}^{P *}>\widetilde{\Pi}^{E *} \Longleftrightarrow \Pi^{P *}>\Pi^{E *} \Longleftrightarrow \gamma>\delta,
$$

so the introduction of $p$ and $d$ does not affect the trade-off determined in Proposition 4 in the main paper. 


\section{Private benefits $(N=1)$}

Revenue is $R(a, e, I)$. The firm derives private benefits $Y(a)$, whereas the agent derives private benefits $y(a)$. We can state an extended version of Proposition 3 in the main paper.

Proposition 1 Suppose $R(a, e, I)$ is supermodular in its arguments, $Y(a)=Y a$ and $y(a)=y a$, where $Y$ and $y$ are positive constants. Then: (i) if $Y>y$ and $t^{P *} \geq 1 / 2$, then $\Pi^{E *}>\Pi^{P *}$; (ii) if $y>Y$ and $t^{E *} \leq 1 / 2$, then $\Pi^{P *}>\Pi^{E *}$.

Proof. For any $\left(\tau_{1}, \tau_{2}, \tau_{3}, \tau_{4}\right) \in[0,1]^{4}$, let

$$
\Pi\left(\tau_{1}, \tau_{2}, \tau_{3}, \tau_{4}\right) \equiv R(a, e, I)+(Y+y) a-c^{a}(a)-c^{e}(e)-c^{I}(I),
$$

where $(a, e, I)$ is the unique solution to the three equations

$$
\begin{aligned}
\tau_{1} R_{a}(a, e, I)+\tau_{2}(Y+y) & =c_{a}^{a}(a) \\
\tau_{3} R_{e}(a, e, I) & =c_{e}^{e}(e) \\
\tau_{4} R_{I}(a, e, I) & =c_{I}^{I}(I)
\end{aligned}
$$

We first prove two preliminary lemmas.

Lemma 1 For all $i \in\{1,2,3,4\}$, the solution $(a, e, I)$ to (6) is strictly increasing in $\tau_{i}$.

Proof. Note that the solution $(a, e, I)$ corresponds to a game in which there are three players, which seek to maximize respectively

$$
\begin{aligned}
f^{1}(a) & \equiv \tau_{1} R(a, e, I)+\tau_{2}(Y+y) a-c^{a}(a) \\
f^{2}(e) & \equiv \tau_{3} R(a, e, I)-c^{e}(e) \\
f^{3}(I) & \equiv \tau_{4} R(a, e, I)-c^{I}(I) .
\end{aligned}
$$

Since $R$ is supermodular, it is easily seen that the game is supermodular with payoffs having strictly increasing differences in the actions $(a, e, I)$ and the parameters $\left(\tau_{1}, \tau_{2}, \tau_{3}, \tau_{4}\right)$. From standard supermodularity results ${ }^{1}$, we know that an increase in any of the parameters $\left(\tau_{1}, \tau_{2}, \tau_{3}, \tau_{4}\right)$ will increase each of the solutions $(a, e, I)$ in a weak sense. To obtain the strict comparative static result, note that the solution is defined by the set of equations defined in (6). This means that if $\tau_{i}$ increases for some

\footnotetext{
${ }^{1}$ See, for instance, X. Vives (1999), Oligopoly Pricing: Old Ideas and New Tools, Cambridge, Mass.: MIT Press.
} 
$i \in\{1,2,3,4\}$ and $a, e$ or $I$ does not strictly increase, then neither $a, e$ and $I$ can change since none can decrease. But if $(a, e, I)$ all remain unchanged, then, since $\tau_{i}$ is higher, the first-order conditions (6) can no longer hold. Thus, at least one among $(a, e, I)$ must strictly increase.

Lemma 2 When $\left(\tau_{1}, \tau_{2}, \tau_{3}, \tau_{4}\right) \in[0,1)^{4}$, the payoff function $\Pi\left(\tau_{1}, \tau_{2}, \tau_{3}, \tau_{4}\right)$ is strictly increasing in $\tau_{i}$ for all $i \in\{1,2,3,4\}$.

Proof. We have

$$
\begin{aligned}
\frac{d \Pi}{d \tau_{1}}= & \left(R_{a}(a, e, I)+Y+y-c_{a}^{a}(a)\right) \frac{d a}{d \tau_{1}} \\
& +\left(R_{e}(a, e, I)-c_{e}^{e}(e)\right) \frac{d e}{d \tau_{1}}+\left(R_{I}(a, e, I)-c_{I}^{I}(I)\right) \frac{d I}{d \tau_{1}} \\
= & \left(\left(1-\tau_{1}\right) R_{a}(a, e, I)+\left(1-\tau_{2}\right)(Y+y)\right) \frac{d a}{d \tau_{1}} \\
& +\left(1-\tau_{3}\right) R_{e}(a, e, I) \frac{d e}{d \tau_{1}}+\left(1-\tau_{3}\right) R_{I}(a, e, I) \frac{d I}{d \tau_{1}},
\end{aligned}
$$

where we have used (6) to replace $c_{a}^{a}(a), c_{e}^{e}(e)$ and $c_{I}^{I}(I)$. By assumption, $R_{a}>0, R_{e}>0$ and $R_{I}>0$, and from Lemma 1 , we know that $\frac{d a}{d \tau_{1}} \geq 0, \frac{d e}{d \tau_{1}} \geq 0$ and $\frac{d I}{d \tau_{1}} \geq 0$, with at least one strict inequality. Thus, we can conclude that $\frac{d \Pi}{d \tau_{1}}>0$ when $\left(\tau_{1}, \tau_{2}, \tau_{3}, \tau_{4}\right) \in[0,1)^{4}$.

A very similar reasoning proves that $\frac{d \Pi}{d \tau_{i}}>0$ for $i=2,3,4$ when $\left(\tau_{1}, \tau_{2}, \tau_{3}, \tau_{4}\right) \in[0,1)^{4}$.

We can now use these lemmas to prove Proposition 1.

Suppose $y>Y$ and $t^{E *} \leq 1 / 2$. Then the firm could strictly improve on $E$-mode profits $\Pi^{E *}$ by giving up control over the transferable action $a$ to the agent and keeping the variable fee unchanged, equal to $t^{E *}$. To see this, note that the change in profits is

$$
\Pi\left(1-t^{E *}, \frac{y}{Y+y}, 1-t^{E *}, t^{E *}\right)-\Pi\left(t^{E *}, \frac{Y}{Y+y}, 1-t^{E *}, t^{E *}\right) .
$$

If $t^{E *}>0$, then this difference is positive by Lemma 2, because $0<t^{E *}<1 / 2$ and $y>Y$ imply $\left(t^{E *}, \frac{Y}{Y+y}, 1-t^{E *}, t^{E *}\right) \in(0,1)^{4}$ and $\left(1-t^{E *}, \frac{y}{Y+y}, 1-t^{E *}, t^{E *}\right)>\left(t^{E *}, \frac{Y}{Y+y}, 1-t^{E *}, t^{E *}\right)$. If $t^{E *}=$ 0 , then the change in profits is

$$
\begin{aligned}
& \Pi\left(1, \frac{y}{Y+y}, 1,0\right)-\Pi\left(0, \frac{Y}{Y+y}, 1,0\right) \\
= & \left(\max _{a, e}\left\{R(a, e, 0)+(Y+y) a-c^{a}(a)-c^{e}(e)\right\} \text { s.t. } R_{a}(a, e, I)+y=c_{a}^{a}(a)\right) \\
& -\max _{e}\left\{R(0, e, 0)-c^{e}(e)\right\},
\end{aligned}
$$


which is positive due to assumptions (a1)-(a4).

Thus, in all cases we have

$$
\Pi^{E *}=\Pi\left(t^{E *}, \frac{Y}{Y+y}, 1-t^{E *}, t^{E *}\right)<\Pi\left(1-t^{E *}, \frac{y}{Y+y}, 1-t^{E *}, t^{E *}\right) \leq \Pi^{P *} .
$$

Similarly, denote by $t^{P *}$ the optimal transaction fee in $E$-mode and suppose $t^{P *}>1 / 2$ and $Y>y$. Then the firm could strictly improve on $P$-mode profits by taking control over the transferable action $a$ and keeping the variable fee unchanged, equal to $t^{P *}$. To see this, note that the change in profits is

$$
\Pi\left(t^{P *}, \frac{Y}{Y+y}, 1-t^{P *}, t^{P *}\right)-\Pi\left(1-t^{P *}, \frac{y}{Y+y}, 1-t^{P *}, t^{P *}\right) .
$$

If $t^{P *}<1$, then this difference is positive by Lemma 2, because $1>t^{P *}>1 / 2$ and $Y>y$ implies $\left(1-t^{P *}, \frac{y}{Y+y}, 1-t^{P *}, t^{P *}\right) \in(0,1)^{4}$ and $\left(t^{P *}, \frac{Y}{Y+y}, 1-t^{P *}, t^{P *}\right)>\left(1-t^{P *}, \frac{y}{Y+y}, 1-t^{P *}, t^{P *}\right)$. If $t^{P *}=1$, then the change in profits is

$$
\begin{aligned}
& \Pi\left(1, \frac{Y}{Y+y}, 0,1\right)-\Pi\left(0, \frac{y}{Y+y}, 0,1\right) \\
= & \left(\max _{a, I}\left\{R(a, 0, I)+(Y+y) a-c^{a}(a)-c^{I}(I)\right\} \text { s.t. } R_{a}(a, e, I)+Y=c_{a}^{a}(a)\right) \\
& -\max _{I}\left\{R(0,0, I)-c^{I}(I)\right\},
\end{aligned}
$$

which is positive due to assumptions (a1)-(a4).

Thus, in all cases we have

$$
\Pi^{P *}=\Pi\left(1-t^{P *}, \frac{y}{Y+y}, 1-t^{P *}, t^{P *}\right)<\Pi\left(t^{P *}, \frac{Y}{Y+y}, 1-t^{P *}, t^{P *}\right)=\Pi^{E *} .
$$

The claim of Proposition 1 says that when the transferable action has a higher impact on the firm's private benefits relative to the agent's, the firm would never find it profitable to function in $P$-mode and charge variable fees above 50\%. Similarly, when the transferable action has a higher impact on the agent's private benefits relative to the firm's, the firm would never find it profitable to function in $E$-mode and pay a bonus above $50 \%$. 
This can be re-stated in a more empirically useful way. To do so, define

$$
t^{*} \equiv\left\{\begin{array}{lll}
t^{E *} & \text { if } \quad \Pi^{E *} \geq \Pi^{P *} \\
t^{P *} & \text { if } \quad \Pi^{E *}<\Pi^{P *}
\end{array}\right.
$$

which is the optimal variable fee charged by the firm in the optimal mode. We obtain the following extension of Corollary 1 in the main paper.

Corollary 1 Suppose $R(a, e, I)$ is supermodular in its arguments, $Y(a)=Y a$ and $y(a)=y a$, where $Y$ and $y$ are positive constants. If $y>Y$ and $t^{*} \leq 1 / 2$, then the P-mode is optimal. If $Y>y$ and $t^{*} \geq 1 / 2$, then the E-mode is optimal.

Thus, if the agent obtains more than $50 \%$ of variable revenues and the agent's private benefits are more important, then the firm should be functioning in $P$-mode, and not in $E$-mode. This suggests (as in Corollary 1 in the main paper), that only observing whether agents keep more or less than $50 \%$ of variable revenue can reveal the mode under which a firm is operating. However, the result requires that the party receiving more than $50 \%$ of variable revenue also has the more important private benefits. In Section 7 below we show numerically that even if this is not the case, knowing whether agents receive more or less than $50 \%$ of variable revenues still allows us to correctly predict the choice of mode most of the time.

\subsection{Linear revenue function and linear private benefits}

Again, we resort to the example with linear revenue function and quadratic cost functions:

$$
\begin{aligned}
R(a, e, I) & =\theta a+\gamma e+\delta I \\
c^{a}(a) & =\frac{1}{2} a^{2}, c^{e}(e)=\frac{1}{2} e^{2}, c^{I}(I)=\frac{1}{2} I^{2} .
\end{aligned}
$$

Private benefits are also assumed to be linear:

$$
Y(a)=Y a \quad \text { and } \quad y(a)=y a
$$

where $Y, y>0$. 
In $E$-mode, the firm solves

$$
\begin{aligned}
\Pi^{E *}= & \max _{t, a, e, I}\left\{(\theta+Y+y) a+\gamma e+\delta I-\frac{1}{2} a^{2}-\frac{1}{2} e^{2}-\frac{1}{2} I^{2}\right\} \\
& \text { subject to } \\
& \left\{\begin{array}{l}
t \theta+Y=a \\
(1-t) \gamma=e \\
t \delta=I
\end{array}\right.
\end{aligned}
$$

Straightforward calculations yield

$$
\begin{aligned}
\Pi^{E *} & =\max _{t}\left\{\frac{1}{2}\left((t \theta+Y)((2-t) \theta+Y+2 y)+\delta^{2} t(2-t)+\gamma^{2}\left(1-t^{2}\right)\right)\right\} \\
& =\max _{t}\left\{\frac{1}{2}\left(2(\theta+Y+y)(t \theta+Y)-(t \theta+Y)^{2}+\delta^{2} t(2-t)+\gamma^{2}\left(1-t^{2}\right)\right)\right\},
\end{aligned}
$$

which leads to

$$
\begin{aligned}
t^{E *} & =\frac{\theta^{2}+\theta y+\delta^{2}}{\theta^{2}+\delta^{2}+\gamma^{2}} \\
\Pi^{E *} & =\frac{1}{2}\left(2 Y(\theta+Y+y)+\gamma^{2}-Y^{2}+\frac{\left(\theta^{2}+\theta y+\delta^{2}\right)^{2}}{\theta^{2}+\delta^{2}+\gamma^{2}}\right)
\end{aligned}
$$

In $P$-mode, the firm solves

$$
\begin{aligned}
\Pi^{P *}= & \max _{t, a, e, I}\left\{(\theta+Y+y) a+\gamma e+\delta I-\frac{1}{2} a^{2}-\frac{1}{2} e^{2}-\frac{1}{2} I^{2}\right\} \\
& \text { subject to } \\
& \left\{\begin{array}{l}
(1-t) \theta+y=a \\
(1-t) \gamma=e \\
t \delta=I
\end{array}\right.
\end{aligned}
$$

Straightforward calculations yield

$$
\begin{aligned}
\Pi^{P *} & =\max _{t}\left\{\frac{1}{2}\left(((1-t) \theta+y)((1+t) \theta+2 Y+y)+\delta^{2} t(2-t)+\gamma^{2}\left(1-t^{2}\right)\right)\right\} \\
& =\max _{t}\left\{\frac{1}{2}\left((\theta+Y+y)^{2}-(t \theta+Y)^{2}+\delta^{2} t(2-t)+\gamma^{2}\left(1-t^{2}\right)\right)\right\},
\end{aligned}
$$


which leads to

$$
\begin{aligned}
t^{P *} & =\frac{\delta^{2}-\theta Y}{\theta^{2}+\delta^{2}+\gamma^{2}} \\
\Pi^{P *} & =\frac{1}{2}\left((\theta+Y+y)^{2}+\gamma^{2}-Y^{2}+\frac{\left(\delta^{2}-\theta Y\right)^{2}}{\theta^{2}+\delta^{2}+\gamma^{2}}\right)
\end{aligned}
$$

Comparing the two profits, the $P$-mode is preferred to the $E$-mode if and only if

$$
\left((\theta+y)^{2}-Y^{2}\right) \gamma^{2}>\left((\theta+Y)^{2}-y^{2}\right) \delta^{2}
$$

\subsection{Linear revenue function and private benefits proportional to demand}

The revenue and cost functions remain the same, but suppose now private benefits are proportional to the demand underlying the linear revenue function:

$$
\begin{aligned}
& Y(a)=Y(\theta a+\gamma e+\delta I) \\
& y(a)=y(\theta a+\gamma e+\delta I),
\end{aligned}
$$

where $\theta a+\gamma e+\delta I$ measures demand and the price is normalized to 1 .

In $E$-mode, the firm solves

$$
\begin{aligned}
\Pi^{E *}= & \max _{t, a, e, I}\left\{(1+Y+y)(\theta a+\gamma e+\delta I)-\frac{1}{2} a^{2}-\frac{1}{2} e^{2}-\frac{1}{2} I^{2}\right\} \\
& \text { subject to } \\
& \left\{\begin{array}{l}
\theta(t+Y)=a \\
\gamma((1-t)+y)=e \\
\delta(t+Y)=I
\end{array}\right.
\end{aligned}
$$

Straightforward calculations yield

$$
\Pi^{E *}=\max _{t}\left\{\frac{1}{2}\left(\left(\theta^{2}+\delta^{2}\right)(t+Y)(2-t+Y+2 y)+\gamma^{2}(1-t+y)(1+t+2 Y+y)\right)\right\}
$$

which leads to

$$
\begin{aligned}
t^{E *} & =\frac{\left(\theta^{2}+\delta^{2}\right)(1+y)-\gamma^{2} Y}{\theta^{2}+\delta^{2}+\gamma^{2}} \\
\Pi^{E *} & =\frac{(1+Y+y)^{2}}{2\left(\theta^{2}+\delta^{2}+\gamma^{2}\right)^{2}}\left(\left(\theta^{2}+\delta^{2}\right)^{2}\left(\theta^{2}+\delta^{2}+2 \gamma^{2}\right)+\gamma^{4}\left(2 \theta^{2}+2 \delta^{2}+\gamma^{2}\right)\right)
\end{aligned}
$$


In $P$-mode, the firm solves

$$
\begin{aligned}
\Pi^{P *}= & \max _{t, a, e, I}\left\{(1+Y+y)(\theta a+\gamma e+\delta I)-\frac{1}{2} a^{2}-\frac{1}{2} e^{2}-\frac{1}{2} I^{2}\right\} \\
& \text { subject to } \\
& \left\{\begin{array}{l}
\theta((1-t)+y)=a \\
\gamma((1-t)+y)=e \\
\delta(t+Y)=I
\end{array}\right.
\end{aligned}
$$

Straightforward calculations yield

$$
\Pi^{P *}=\max _{t}\left\{\frac{1}{2}\left(\delta^{2}(t+Y)(2-t+Y+2 y)+\left(\theta^{2}+\gamma^{2}\right)(1-t+y)(1+t+2 Y+y)\right)\right\},
$$

which leads to

$$
\begin{aligned}
t^{P *} & =\frac{\delta^{2}(1+y)-\left(\theta^{2}+\gamma^{2}\right) Y}{\theta^{2}+\delta^{2}+\gamma^{2}} \\
\Pi^{P *} & =\frac{(1+Y+y)^{2}}{2\left(\theta^{2}+\delta^{2}+\gamma^{2}\right)^{2}}\left(\delta^{4}\left(2 \theta^{2}+\delta^{2}+2 \gamma^{2}\right)+\left(\theta^{2}+\gamma^{2}\right)^{2}\left(\theta^{2}+2 \delta^{2}+\gamma^{2}\right)\right)
\end{aligned}
$$

Comparing the two profits, the $P$-mode dominates the $E$-mode if and only if

$$
\begin{aligned}
\delta^{4}\left(2 \theta^{2}+\delta^{2}+2 \gamma^{2}\right)+\left(\theta^{2}+\gamma^{2}\right)^{2}\left(\theta^{2}+2 \delta^{2}+\gamma^{2}\right) & >\left(\theta^{2}+\delta^{2}\right)^{2}\left(\theta^{2}+\delta^{2}+2 \gamma^{2}\right)+\gamma^{4}\left(2 \theta^{2}+2 \delta^{2}+\gamma^{2}\right) \\
\delta^{4}+\left(\theta^{2}+\gamma^{2}\right)^{2}+\delta^{2}\left(\theta^{2}+\gamma^{2}\right) & >\left(\theta^{2}+\delta^{2}\right)^{2}+\gamma^{4}+\left(\theta^{2}+\delta^{2}\right) \gamma^{2} \\
2 \theta^{2} \gamma^{2}+\delta^{2}\left(\theta^{2}+\gamma^{2}\right) & >2 \theta^{2} \delta^{2}+\left(\theta^{2}+\delta^{2}\right) \gamma^{2} \\
\gamma^{2} & >\delta^{2}
\end{aligned}
$$

Thus, with this specification of private benefits, they have no impact on the trade-off between $E$-mode and $P$-mode. This result might seem surprising, particularly in the case when $Y>0$ and $y=0$ : one would then expect the $E$-mode to always dominate because the firm takes the private benefit $Y$ into account when setting $a$, whereas the agent does not. The reason this is not true is that in $P$-mode the firm needs to provide the agent with sufficient incentives for choosing both $a$ and $e$, so it sets $t^{P *}$ sufficiently low (specifically, $1-t^{P *}>t^{E *}+Y$ ) such that it compensates for the fact that the agent does not internalize the firm's private $Y$ when setting $a$. 


\section{Cost asymmetries}

Consider the linear example with one agent

$$
R(a, e, I)=\theta a+\gamma e+\delta I
$$

and allow for different costs of the transferable action in the two modes:

$$
c_{E}^{a}(a)=\frac{c_{E}}{2} a^{2}, \quad c_{P}^{a}(a)=\frac{c_{P}}{2} a^{2}, \quad c^{e}(e)=\frac{1}{2} e^{2} \quad \text { and } \quad c^{I}(I)=\frac{1}{2} I^{2} .
$$

Let then ${ }^{2}$

$$
\theta_{E} \equiv \frac{\theta}{\sqrt{c_{E}}} \quad \text { and } \quad \theta_{P} \equiv \frac{\theta}{\sqrt{c_{P}}}
$$

Straightforward calculations lead to

$$
\begin{aligned}
\Pi^{E *} & =\max _{t}\left\{\frac{1}{2}\left(\left(\theta_{E}^{2}+\delta^{2}\right) t(2-t)+\gamma^{2}\left(1-t^{2}\right)\right)\right\} \\
t^{E *} & =\frac{\theta_{E}^{2}+\delta^{2}}{\theta_{E}^{2}+\delta^{2}+\gamma^{2}}
\end{aligned}
$$

and

$$
\begin{aligned}
\Pi^{P *} & =\max _{t}\left\{\frac{1}{2}\left(\delta^{2} t(2-t)+\left(\theta_{P}^{2}+\gamma^{2}\right)\left(1-t^{2}\right)\right)\right\} \\
t^{P *} & =\frac{\delta^{2}}{\theta_{P}^{2}+\delta^{2}+\gamma^{2}} .
\end{aligned}
$$

Let us determine the effects of $\gamma$ and $\delta$ on the tradeoff between the two modes. Using the envelope theorem, we obtain

$$
\begin{aligned}
& \frac{d\left(\Pi^{P *}-\Pi^{E *}\right)}{d\left(\gamma^{2}\right)}>0 \text { if and only if } t^{P *}<t^{E *} \\
& \frac{d\left(\Pi^{P *}-\Pi^{E *}\right)}{d\left(\delta^{2}\right)}<0 \text { if and only if } t^{P *}<t^{E *} .
\end{aligned}
$$

And we have

$$
t^{E *}=\frac{\theta_{E}^{2}+\delta^{2}}{\theta_{E}^{2}+\delta^{2}+\gamma^{2}}>\frac{\delta^{2}}{\delta^{2}+\gamma^{2}}>\frac{\delta^{2}}{\theta_{P}^{2}+\delta^{2}+\gamma^{2}}=t^{P *} .
$$

Therefore, the magnitudes of moral hazard $\gamma$ and $\delta$ have the expected effect on the trade-off.

\footnotetext{
${ }^{2}$ We could also allow for the impact $\theta$ of the transferable action on revenue to differ depending on mode: $\theta^{E}$ and $\theta^{P}$. This would have the exact same effect as the difference in cost.
} 


\section{Example with price as transferable decision and linear demand}

Recall the revenue function

$$
R_{i}\left(p_{i}, \bar{p}_{-i}, e_{i}, I\right)=p_{i}\left(d+\theta p_{i}+x\left(\bar{p}_{-i}-p_{i}\right)+\gamma e_{i}+\delta I\right)
$$

and the assumptions made on parameters in the main paper:

$$
\begin{aligned}
\theta & <0, \gamma>0, \delta>0 \\
-2 \theta+\min \{0,2 x\} & >\max \left\{N \delta^{2}, \gamma^{2}\right\} .
\end{aligned}
$$

The fixed costs of agents' effort and firm's investment are quadratic:

$$
c_{i}^{e}(e)=\frac{1}{2} e^{2}, \quad c^{I}(I)=\frac{1}{2} I^{2} .
$$

In $E$-mode, the payoff to agent $i$ from working for the firm is

$$
(1-t) R_{i}\left(p_{i}, \bar{p}_{-i}, e_{i}, I\right)-\frac{1}{2} e_{i}^{2}-T=(1-t) p_{i}\left(d+\theta p_{i}+x\left(\bar{p}_{-i}-p_{i}\right)+\gamma e_{i}+\delta I\right)-\frac{1}{2} e_{i}^{2}-T,
$$

which the agent optimizes over $e_{i}$ in the second stage (the fixed fee $T$ is then taken as fixed).

The firm's payoff in the second stage is

$$
\sum_{i=1}^{N}\left(t p_{i}\left(d+\theta p_{i}+x\left(\bar{p}_{-i}-p_{i}\right)+\gamma e_{i}+\delta I\right)\right)-\frac{1}{2} I^{2}
$$

which the firm optimizes over $p_{i}$ and $I$.

Evaluating the corresponding first-order conditions at the symmetric equilibrium, we have

$$
\left\{\begin{array}{l}
-2 \theta p^{E}=d+\gamma e^{E}+\delta I^{E} \\
e^{E}=(1-t) \gamma p^{E} \\
I^{E}=t N \delta p^{E}
\end{array}\right.
$$

Solving, we obtain

$$
\begin{aligned}
p^{E}(t) & =\frac{d}{-2 \theta-(1-t) \gamma^{2}-t N \delta^{2}} \\
e^{E}(t) & =\frac{d(1-t) \gamma}{-2 \theta-(1-t) \gamma^{2}-t N \delta^{2}} \\
I^{E}(t) & =\frac{d t N \delta}{-2 \theta-(1-t) \gamma^{2}-t N \delta^{2}} .
\end{aligned}
$$


Note that assumptions $(7)$ ensure $p^{E}(t)>0, e^{E}(t)>0$ and $I^{E}(t)>0$.

The fixed fee $T$ is just a transfer that renders each employee indifferent between working for the firm and their outside option, so the firm's profit is

$$
\Pi^{E}(t)=N p^{E}(t)\left(d+\theta p^{E}(t)+\gamma e^{E}(t)+\delta I^{E}(t)\right)-N \frac{1}{2} e^{E}(t)^{2}-\frac{1}{2} I^{E}(t)^{2} .
$$

Plugging in the expressions of $p^{E}(t), e^{E}(t)$ and $I^{E}(t)$ above, we obtain:

$$
\Pi^{E}(t)=\max _{t}\left\{\frac{N d^{2}\left(-2 \theta-(1-t)^{2} \gamma^{2}-t^{2} N \delta^{2}\right)}{2\left(-2 \theta-(1-t) \gamma^{2}-t N \delta^{2}\right)^{2}}\right\}
$$

In $P$-mode, an agent joining the platform chooses $\left(p_{i}, e_{i}\right)$ to maximize his second stage payoff

$$
(1-t) p_{i}\left(d+\theta p_{i}+x\left(\bar{p}_{-i}-p_{i}\right)+\gamma e_{i}+\delta I\right)-\frac{1}{2} e_{i}^{2}
$$

while the firm chooses $I$ to maximize its second stage revenues

$$
\sum_{i=1}^{N} t p_{i}\left(d+\theta p_{i}+x\left(\bar{p}_{-i}-p_{i}\right)+\gamma e_{i}+\delta I\right)-\frac{1}{2} I^{2}
$$

Evaluating the corresponding first-order conditions at the symmetric equilibrium, we have

$$
\left\{\begin{array}{l}
(-2 \theta+x) p^{P}=d+\gamma e^{P}+\delta I^{P} \\
e^{P}=(1-t) \gamma p^{P} \\
I^{P}=t N \delta p^{P} .
\end{array}\right.
$$

Solving, we obtain:

$$
\begin{aligned}
p^{P}(t) & =\frac{d}{-2 \theta+x-(1-t) \gamma^{2}-t N \delta^{2}} \\
e^{P}(t) & =\frac{d(1-t) \gamma}{-2 \theta+x-(1-t) \gamma^{2}-t N \delta^{2}} \\
I^{P}(t) & =\frac{d t N \delta}{-2 \theta+x-(1-t) \gamma^{2}-t N \delta^{2}} .
\end{aligned}
$$

Assumptions (7) ensure $p^{P}(t)>0, e^{P}(t)>0$ and $I^{P}(t)>0$.

The fixed fee $T$ renders each agent indifferent between joining the platform and his outside option, so the firm's profit in $P$-mode is

$$
\Pi^{P}(t)=N p^{P}(t)\left(d+\theta p^{P}(t)+\gamma e^{P}(t)+\delta I^{P}(t)\right)-N \frac{1}{2} e^{P}(t)^{2}-\frac{1}{2} I^{P}(t)^{2} .
$$


Plugging in the expressions of $p^{P}(t), e^{P}(t)$ and $I^{P}(t)$ above, we obtain:

$$
\Pi^{P}(t)=\max _{t}\left\{\frac{N d^{2}\left(2(-\theta+x)-(1-t)^{2} \gamma^{2}-t^{2} N \delta^{2}\right)}{2\left(-2 \theta+x-(1-t) \gamma^{2}-t N \delta^{2}\right)^{2}}\right\}
$$

Comparing expressions (8) and $(9), \Pi^{E}(t)$ is obtained from $\Pi^{P}(t)$ simply by setting $x=0$. Therefore, we will focus on maximizing $\Pi^{P}(t)$, from which we can easily derive the maximization of $\Pi^{E}(t)$.

The first-order derivative of $\Pi^{P}(t)$ in $t$ is proportional to (with a strictly positive multiplying factor)

$$
N \delta^{2}(-2 \theta+2 x)-\gamma^{2} x-N \delta^{2} \gamma^{2}-t\left(\left(N \delta^{2}+\gamma^{2}\right)(-2 \theta+x)-2 N \delta^{2} \gamma^{2}\right)
$$

Since $\left(N \delta^{2}+\gamma^{2}\right)(-2 \theta+x)-2 N \delta^{2} \gamma^{2}>0$ under assumptions (7), we obtain that the optimal variable fee under the $P$-mode is

$$
t^{P *}=\left\{\begin{array}{cc}
0 & \text { if } N \delta^{2}(-2 \theta+2 x)-\gamma^{2} x-N \delta^{2} \gamma^{2} \leq 0 \\
\frac{N \delta^{2}(-2 \theta+2 x)-\gamma^{2} x-N \delta^{2} \gamma^{2}}{\left(N \delta^{2}+\gamma^{2}\right)(-2 \theta+x)-2 N \delta^{2} \gamma^{2}} & \text { if } 0 \leq N \delta^{2}(-2 \theta+2 x)-\gamma^{2} x-N \delta^{2} \gamma^{2} \\
& \leq\left(N \delta^{2}+\gamma^{2}\right)(-2 \theta+x)-2 N \delta^{2} \gamma^{2} \\
1 & \text { if } N \delta^{2}(-2 \theta+2 x)-\gamma^{2} x-N \delta^{2} \gamma^{2} \\
& \geq\left(N \delta^{2}+\gamma^{2}\right)(-2 \theta+x)-2 N \delta^{2} \gamma^{2}
\end{array}\right.
$$

Rewriting the conditions:

$$
t^{P *}=\left\{\begin{array}{cc}
0 & \text { if } x\left(\gamma^{2}-2 N \delta^{2}\right) \geq N \delta^{2}\left(-2 \theta-\gamma^{2}\right) \\
\frac{N \delta^{2}(-2 \theta+2 x)-\gamma^{2} x-N \delta^{2} \gamma^{2}}{\left(N \delta^{2}+\gamma^{2}\right)(-2 \theta+x)-2 N \delta^{2} \gamma^{2}} & \text { if } x\left(\gamma^{2}-2 N \delta^{2}\right) \leq N \delta^{2}\left(-2 \theta-\gamma^{2}\right) \\
1 & \text { and } x\left(N \delta^{2}-2 \gamma^{2}\right) \leq \gamma^{2}\left(-2 \theta-N \delta^{2}\right) \\
1 & \text { if } x\left(N \delta^{2}-2 \gamma^{2}\right) \geq \gamma^{2}\left(-2 \theta-N \delta^{2}\right) .
\end{array}\right.
$$

Suppose $x$ is such that $0<t^{P *}<1$. Then the first-order condition of $\Pi^{P}(t)$ in $t$ evaluated at $t^{P *}$ implies:

$$
\left(\left(1-t^{P *}\right) \gamma^{2}-t^{P *} N \delta^{2}\right)\left(\begin{array}{c}
-2 \theta+x-\left(1-t^{P *}\right) \gamma^{2} \\
-t^{P *} N \delta^{2}
\end{array}\right)=\left(\gamma^{2}-N \delta^{2}\right)\left(\begin{array}{c}
2(-\theta+x)-\left(1-t^{P *}\right)^{2} \gamma^{2} \\
-\left(t^{P *}\right)^{2} N \delta^{2}
\end{array}\right)
$$


from which we can deduce:

$$
\begin{aligned}
\Pi^{P} & =\frac{N d^{2}\left(2(-\theta+x)-\left(1-t^{P *}\right)^{2} \gamma^{2}-\left(t^{P *}\right)^{2} N \delta^{2}\right)}{2\left(-2 \theta+x-\left(1-t^{P *}\right) \gamma^{2}-t^{P *} N \delta^{2}\right)^{2}} \\
& =\frac{N d^{2}\left(\left(1-t^{P *}\right) \gamma^{2}-t^{P *} N \delta^{2}\right)}{2\left(\gamma^{2}-N \delta^{2}\right)\left(-2 \theta+x-\left(1-t^{P *}\right) \gamma^{2}-t^{P *} N \delta^{2}\right)} \\
& =\frac{N d^{2}}{2\left(\gamma^{2}-N \delta^{2}\right)} \frac{\gamma^{2}-t^{P *}\left(N \delta^{2}+\gamma^{2}\right)}{-2 \theta+x-\gamma^{2}+t^{P *}\left(\gamma^{2}-N \delta^{2}\right)} .
\end{aligned}
$$

Plugging $t^{P *}=\frac{N \delta^{2}(-2 \theta+2 x)-\gamma^{2} x-N \delta^{2} \gamma^{2}}{\left(N \delta^{2}+\gamma^{2}\right)(-2 \theta+x)-2 N \delta^{2} \gamma^{2}}$ into the last expression, we obtain

$$
\Pi^{P *}=\frac{N d^{2}}{2} \frac{(-2 \theta+2 x)\left(N \delta^{2}+\gamma^{2}\right)-N \delta^{2} \gamma^{2}}{\left(N \delta^{2}+\gamma^{2}\right)\left(-2 \theta-N \delta^{2}+x\right)\left(-2 \theta-\gamma^{2}+x\right)-x\left(N \delta^{2}-\gamma^{2}\right)^{2}} .
$$

From here, we can set $x=0$ to obtain

$$
\begin{aligned}
t^{E *} & =\frac{\left(-2 \theta-\gamma^{2}\right) N \delta^{2}}{-2 \theta\left(N \delta^{2}+\gamma^{2}\right)-2 N \delta^{2} \gamma^{2}} \in(0,1) \\
\Pi^{E *} & =\frac{N d^{2}}{2} \frac{-2 \theta\left(N \delta^{2}+\gamma^{2}\right)-N \delta^{2} \gamma^{2}}{\left(N \delta^{2}+\gamma^{2}\right)\left(-2 \theta-N \delta^{2}\right)\left(-2 \theta-\gamma^{2}\right)} .
\end{aligned}
$$

The complete characterization of profits in $P$-mode is:

$$
\Pi^{P *}=\left\{\begin{array}{cc}
\frac{N d^{2}}{2} \frac{2(-\theta+x)-\gamma^{2}}{\left(-2 \theta+x-\gamma^{2}\right)^{2}} & \text { if } x\left(\gamma^{2}-2 N \delta^{2}\right) \geq N \delta^{2}\left(-2 \theta-\gamma^{2}\right) \\
\frac{(-2 \theta+2 x)\left(N \delta^{2}+\gamma^{2}\right)-N \delta^{2} \gamma^{2}}{2} \frac{\text { if } x\left(\gamma^{2}-2 N \delta^{2}\right) \leq N \delta^{2}\left(-2 \theta-\gamma^{2}\right)}{\left(N \delta^{2}+\gamma^{2}\right)\left(-2 \theta-N \delta^{2}+x\right)\left(-2 \theta-\gamma^{2}+x\right)-x\left(N \delta^{2}-\gamma^{2}\right)^{2}} & \text { and } x\left(N \delta^{2}-2 \gamma^{2}\right) \leq \gamma^{2}\left(-2 \theta-N \delta^{2}\right) \\
\frac{N d^{2}}{2} \frac{2(-\theta+x)-N \delta^{2}}{\left(-2 \theta+x-N \delta^{2}\right)^{2}} & \text { if } x\left(N \delta^{2}-2 \gamma^{2}\right) \geq \gamma^{2}\left(-2 \theta-N \delta^{2}\right) .
\end{array}\right.
$$

Suppose $x\left(\gamma^{2}-2 N \delta^{2}\right) \leq N \delta^{2}\left(-2 \theta-\gamma^{2}\right)$ and $x\left(N \delta^{2}-2 \gamma^{2}\right) \leq \gamma^{2}\left(-2 \theta-N \delta^{2}\right)$, so that $0<$ $t^{P *}<1$. We have $\Pi^{P}>\Pi^{E}$ if and only if

$$
\frac{(-2 \theta+2 x)\left(N \delta^{2}+\gamma^{2}\right)-N \delta^{2} \gamma^{2}}{\left(N \delta^{2}+\gamma^{2}\right)\left(-2 \theta-N \delta^{2}+x\right)\left(-2 \theta-\gamma^{2}+x\right)-x\left(N \delta^{2}-\gamma^{2}\right)^{2}}>\frac{-2 \theta\left(N \delta^{2}+\gamma^{2}\right)-N \delta^{2} \gamma^{2}}{\left(N \delta^{2}+\gamma^{2}\right)\left(-2 \theta-N \delta^{2}\right)\left(-2 \theta-\gamma^{2}\right)}
$$

which is equivalent to

$$
\begin{aligned}
& \left((-2 \theta+2 x)\left(N \delta^{2}+\gamma^{2}\right)-N \delta^{2} \gamma^{2}\right)\left(N \delta^{2}+\gamma^{2}\right)\left(-2 \theta-N \delta^{2}\right)\left(-2 \theta-\gamma^{2}\right) \\
> & \left(-2 \theta\left(N \delta^{2}+\gamma^{2}\right)-N \delta^{2} \gamma^{2}\right)\left(\left(N \delta^{2}+\gamma^{2}\right)\left(-2 \theta-N \delta^{2}+x\right)\left(-2 \theta-\gamma^{2}+x\right)-x\left(N \delta^{2}-\gamma^{2}\right)^{2}\right) .
\end{aligned}
$$

Recall the two sides are equal for $x=0$, therefore we can eliminate all terms that are not factored by 
$x$ or $x^{2}$, so the inequality reduces to

$$
\begin{aligned}
& 2 x\left(N \delta^{2}+\gamma^{2}\right)^{2}\left(-2 \theta-N \delta^{2}\right)\left(-2 \theta-\gamma^{2}\right) \\
> & \left(-2 \theta\left(N \delta^{2}+\gamma^{2}\right)-N \delta^{2} \gamma^{2}\right)\left(-x\left(N \delta^{2}-\gamma^{2}\right)^{2}+x\left(N \delta^{2}+\gamma^{2}\right)\left(-4 \theta-\left(N \delta^{2}+\gamma^{2}\right)\right)+x^{2}\left(N \delta^{2}+\gamma^{2}\right)\right) .
\end{aligned}
$$

Rearranging, this can be rewritten

$$
\begin{aligned}
0> & -x\left(\begin{array}{c}
\left(-2 \theta\left(N \delta^{2}+\gamma^{2}\right)-N \delta^{2} \gamma^{2}\right)\left(2\left(N^{2} \delta^{4}+\gamma^{4}\right)+4 \theta\left(N \delta^{2}+\gamma^{2}\right)\right) \\
+2\left(N \delta^{2}+\gamma^{2}\right)^{2}\left(-2 \theta-N \delta^{2}\right)\left(-2 \theta-\gamma^{2}\right)
\end{array}\right)+ \\
& +x^{2}\left(-2 \theta\left(N \delta^{2}+\gamma^{2}\right)-N \delta^{2} \gamma^{2}\right)\left(N \delta^{2}+\gamma^{2}\right) .
\end{aligned}
$$

Simplifying, this leads to

$$
0>-2 x N \delta^{2} \gamma^{2}\left(2 \theta\left(N \delta^{2}+\gamma^{2}\right)+2 N \delta^{2} \gamma^{2}\right)+x^{2}\left(-2 \theta\left(N \delta^{2}+\gamma^{2}\right)-N \delta^{2} \gamma^{2}\right)\left(N \delta^{2}+\gamma^{2}\right),
$$

from which we conclude

$$
\Pi^{P *}>\Pi^{E *} \Longleftrightarrow x\left(\frac{2 N \delta^{2} \gamma^{2}\left(-2 \theta\left(N \delta^{2}+\gamma^{2}\right)-2 N \delta^{2} \gamma^{2}\right)}{\left(-2 \theta\left(N \delta^{2}+\gamma^{2}\right)-N \delta^{2} \gamma^{2}\right)\left(N \delta^{2}+\gamma^{2}\right)}+x\right)<0
$$

Both the numerator and the denominator of the large fraction are positive under assumptions (7).

We conclude that when $0<t^{P *}<1$ :

$$
\begin{aligned}
\Pi^{P *} & >\quad \Pi^{E *} \Longleftrightarrow-\frac{2 N \delta^{2} \gamma^{2}\left(-2 \theta\left(N \delta^{2}+\gamma^{2}\right)-2 N \delta^{2} \gamma^{2}\right)}{\left(-2 \theta\left(N \delta^{2}+\gamma^{2}\right)-N \delta^{2} \gamma^{2}\right)\left(N \delta^{2}+\gamma^{2}\right)}<x<0 \\
& \Longleftrightarrow \frac{-4\left(\theta \frac{N \delta^{2}+\gamma^{2}}{N \delta^{2} \gamma^{2}}+1\right)}{\frac{N \delta^{2}+\gamma^{2}}{N \delta^{2} \gamma^{2}}\left(2 \theta \frac{N \delta^{2}+\gamma^{2}}{N \delta^{2} \gamma^{2}}+1\right)}<x<0 .
\end{aligned}
$$

It remains to consider the cases $x\left(\gamma^{2}-2 N \delta^{2}\right) \geq N \delta^{2}\left(-2 \theta-\gamma^{2}\right)\left(\right.$ in which $\left.t^{P *}=0\right)$ and $x\left(N \delta^{2}-2 \gamma^{2}\right) \geq$ $\gamma^{2}\left(-2 \theta-N \delta^{2}\right)$ (in which $t^{P *}=1$ ). It is easier to consider the following three cases in turn.

Case I: $\gamma^{2}>2 N \delta^{2}$.

In this case, it is easily verified that assumptions (7) imply $x\left(N \delta^{2}-2 \gamma^{2}\right) \leq \gamma^{2}\left(-2 \theta-N \delta^{2}\right)$. Therefore we have:

$$
\Pi^{P *}=\left\{\begin{array}{cc}
\frac{N d^{2}}{2} \frac{2(-\theta+x)-\gamma^{2}}{\left(-2 \theta+x-\gamma^{2}\right)^{2}} & \text { if } x \geq \frac{N \delta^{2}\left(-2 \theta-\gamma^{2}\right)}{\gamma^{2}-2 N \delta^{2}} \\
\frac{(-2 \theta+2 x)\left(N \delta^{2}+\gamma^{2}\right)-N \delta^{2} \gamma^{2}}{2} \frac{\left(N \delta^{2}+\gamma^{2}\right)\left(-2 \theta-N \delta^{2}+x\right)\left(-2 \theta-\gamma^{2}+x\right)-x\left(N \delta^{2}-\gamma^{2}\right)^{2}}{(f)} \frac{N \delta^{2}\left(-2 \theta-\gamma^{2}\right)}{\gamma^{2}-2 N \delta^{2}} \geq x \geq-\frac{-2 \theta-\max \left\{\gamma^{2}, N \delta^{2}\right\}}{2} .
\end{array}\right.
$$


The expression $\frac{2(-\theta+x)-\gamma^{2}}{\left(-2 \theta+x-\gamma^{2}\right)^{2}}$ is increasing in $x$ for $x \leq 0$ and decreasing in $x$ for $x \geq 0$, therefore the maximum value attained by $\Pi^{P}$ when $x \geq \frac{N \delta^{2}\left(-2 \theta-\gamma^{2}\right)}{\gamma^{2}-2 N \delta^{2}}$ is precisely when $x=\frac{N \delta^{2}\left(-2 \theta-\gamma^{2}\right)}{\gamma^{2}-2 N \delta^{2}}$. That value is:

$$
\begin{aligned}
\Pi^{P *}\left(x=\frac{N \delta^{2}\left(-2 \theta-\gamma^{2}\right)}{\gamma^{2}-2 N \delta^{2}}\right) & =\frac{N d^{2}}{2} \frac{\gamma^{2}\left(\gamma^{2}-2 N \delta^{2}\right)}{\left(-2 \theta-\gamma^{2}\right)\left(\gamma^{2}-N \delta^{2}\right)^{2}} \\
& <\frac{N d^{2}}{2} \frac{(-2 \theta)\left(N \delta^{2}+\gamma^{2}\right)-N \delta^{2} \gamma^{2}}{\left(N \delta^{2}+\gamma^{2}\right)\left(-2 \theta-N \delta^{2}\right)\left(-2 \theta-\gamma^{2}\right)}=\Pi^{E *}
\end{aligned}
$$

where the inequality is straightforward to verify under assumptions (7). Thus, $\Pi^{E *}$ dominates $\Pi^{P *}$ for all $x \geq \frac{N \delta^{2}\left(-2 \theta-\gamma^{2}\right)}{\gamma^{2}-2 N \delta^{2}}$. Combining with the result above, we conclude that $\Pi^{E *}$ dominates $\Pi^{P *}$ for all $x \geq 0$ and $x \leq \frac{-4\left(\theta \frac{N \delta^{2}+\gamma^{2}}{N \delta^{2} \gamma^{2}}+1\right)}{\frac{N \delta^{2}+\gamma^{2}}{N \delta^{2} \gamma^{2}}\left(2 \theta \frac{N \delta^{2}+\gamma^{2}}{N \delta^{2} \gamma^{2}}+1\right)}$, whereas $\Pi^{P *}$ dominates $\Pi^{E *}$ for all permissible $x$ such that

$$
\frac{-4\left(\theta \frac{N \delta^{2}+\gamma^{2}}{N \delta^{2} \gamma^{2}}+1\right)}{\frac{N \delta^{2}+\gamma^{2}}{N \delta^{2} \gamma^{2}}\left(2 \theta \frac{N \delta^{2}+\gamma^{2}}{N \delta^{2} \gamma^{2}}+1\right)} \leq x \leq 0
$$

Case II: $N \delta^{2}>2 \gamma^{2}$.

In this case, it is easily verified that assumptions (7) imply $x\left(\gamma^{2}-2 N \delta^{2}\right) \leq N \delta^{2}\left(-2 \theta-\gamma^{2}\right)$. Therefore we have:

$$
\Pi^{P *}=\left\{\begin{array}{cc}
\frac{N d^{2}}{2} \frac{2(-\theta+x)-N \delta^{2}}{\left(-2 \theta+x-N \delta^{2}\right)^{2}} & \text { if } x \geq \frac{\gamma^{2}\left(-2 \theta-N \delta^{2}\right)}{N \delta^{2}-2 \gamma^{2}} \\
\frac{(-2 \theta+2 x)\left(N \delta^{2}+\gamma^{2}\right)-N \delta^{2} \gamma^{2}}{2} \frac{N d^{2}}{\left(N \delta^{2}+\gamma^{2}\right)\left(-2 \theta-N \delta^{2}+x\right)\left(-2 \theta-\gamma^{2}+x\right)-x\left(N \delta^{2}-\gamma^{2}\right)^{2}} & \text { if } \frac{\gamma^{2}\left(-2 \theta-N \delta^{2}\right)}{N \delta^{2}-2 \gamma^{2}} \geq x \geq-\frac{-2 \theta-\max \left\{\gamma^{2}, N \delta^{2}\right\}}{2} .
\end{array}\right.
$$

The analysis is exactly the same as in Case I above (by symmetry in $\gamma^{2}$ and $N \delta^{2}$ ), therefore the conclusion is exactly the same for this case as well.

Case III: $\gamma^{2} \leq 2 N \delta^{2}$ and $N \delta^{2} \leq 2 \gamma^{2}$.

In this case, it is easily verified that assumptions (7) imply $x\left(N \delta^{2}-2 \gamma^{2}\right) \leq \gamma^{2}\left(-2 \theta-N \delta^{2}\right)$ and $x\left(\gamma^{2}-2 N \delta^{2}\right) \leq N \delta^{2}\left(-2 \theta-\gamma^{2}\right)$ for all permissible $x$. Therefore we have:

$$
\Pi^{P *}=\frac{N d^{2}}{2} \frac{(-2 \theta+2 x)\left(N \delta^{2}+\gamma^{2}\right)-N \delta^{2} \gamma^{2}}{\left(N \delta^{2}+\gamma^{2}\right)\left(-2 \theta-N \delta^{2}+x\right)\left(-2 \theta-\gamma^{2}+x\right)-x\left(N \delta^{2}-\gamma^{2}\right)^{2}}
$$


for all permissible $x$, so we already know that

$$
\Pi^{P *}>\Pi^{E *} \Longleftrightarrow \frac{-4\left(\theta \frac{N \delta^{2}+\gamma^{2}}{N \delta^{2} \gamma^{2}}+1\right)}{\frac{N \delta^{2}+\gamma^{2}}{N \delta^{2} \gamma^{2}}\left(2 \theta \frac{N \delta^{2}+\gamma^{2}}{N \delta^{2} \gamma^{2}}+1\right)}<x<0 .
$$

\section{Hybrid mode across agents}

Recall the expression of firm profits when using a hybrid mode as a function of $\left(t^{E}, t^{P}, n\right)$ :

$$
\begin{aligned}
\Pi^{H}\left(t^{E}, t^{P}, n\right)= & n\left(\frac{t^{E}\left(2-t^{E}\right) \theta^{2}}{2}+\frac{\left(1-\left(t^{E}\right)^{2}\right) \gamma^{2}}{2}\right)+(N-n)\left(\frac{\left(1-\left(t^{P}\right)^{2}\right) \theta^{2}}{2}+\frac{\left(1-\left(t^{P}\right)^{2}\right) \gamma^{2}}{2}\right) \\
& +\frac{\bar{t}(2-\bar{t}) N^{2} \delta^{2}}{2},
\end{aligned}
$$

where

$$
\bar{t} \equiv \frac{n}{N} t^{E}+\frac{N-n}{N} t^{P} .
$$

Optimizing over the three variables $\left(t^{E}, t^{P}, n\right)$ yields the following first-order conditions (assuming interior solution in all three variables):

$$
\left\{\begin{array}{l}
\theta^{2}+N \delta^{2}-\left(\theta^{2}+\gamma^{2}+n \delta^{2}\right) t^{E}-(N-n) \delta^{2} t^{P}=0 \\
N \delta^{2}-n \delta^{2} t^{E}-\left(\theta^{2}+\gamma^{2}+(N-n) \delta^{2}\right) t^{P}=0 \\
\frac{\theta^{2}}{2}\left(t^{E}\left(2-t^{E}\right)-1+\left(t^{P}\right)^{2}\right)+\frac{\gamma^{2}}{2}\left(\left(t^{P}\right)^{2}-\left(t^{E}\right)^{2}\right)+N \delta^{2}(1-\bar{t})\left(t^{E}-t^{P}\right)=0 .
\end{array}\right.
$$

Solving the first two first-order conditions above for $\left(t^{E}, t^{P}\right)$ as functions of $n$, we obtain:

$$
\begin{aligned}
t^{E} & =\frac{\left(\theta^{2}+N \delta^{2}\right)\left(\theta^{2}+\gamma^{2}\right)+(N-n) \delta^{2} \theta^{2}}{\left(\theta^{2}+\gamma^{2}\right)\left(\theta^{2}+\gamma^{2}+N \delta^{2}\right)} \\
t^{P} & =\frac{(N-n) \delta^{2} \theta^{2}+N \delta^{2} \gamma^{2}}{\left(\theta^{2}+\gamma^{2}\right)\left(\theta^{2}+\gamma^{2}+N \delta^{2}\right)} .
\end{aligned}
$$

This implies:

$$
\begin{aligned}
& t^{E}-t^{P}=\frac{\theta^{2}}{\theta^{2}+\gamma^{2}} \\
& N(1-\bar{t})=\frac{(N-n) \theta^{2}+N \gamma^{2}}{\theta^{2}+\gamma^{2}+N \delta^{2}} .
\end{aligned}
$$


We can now plug these expressions in the third first-order condition above, which becomes:

$$
\begin{aligned}
\frac{\theta^{2}}{2}\left(t^{E}\left(2-t^{E}\right)-1+\left(t^{P}\right)^{2}\right)+\frac{\gamma^{2}}{2}\left(\left(t^{P}\right)^{2}-\left(t^{E}\right)^{2}\right)+\frac{\theta^{2} \delta^{2}}{\theta^{2}+\gamma^{2}} \frac{(N-n) \theta^{2}+N \gamma^{2}}{\theta^{2}+\gamma^{2}+N \delta^{2}} & =0 \\
\frac{\theta^{2}}{2}\left(2 t^{E}-1\right)-\frac{\theta^{2}+\gamma^{2}}{2} \frac{\theta^{2}}{\theta^{2}+\gamma^{2}}\left(t^{E}+t^{P}\right)+\frac{\theta^{2} \delta^{2}}{\theta^{2}+\gamma^{2}} \frac{(N-n) \theta^{2}+N \gamma^{2}}{\theta^{2}+\gamma^{2}+N \delta^{2}} & =0 \\
\frac{1}{2}\left(t^{E}-t^{P}-1\right)+\frac{\delta^{2}}{\theta^{2}+\gamma^{2}} \frac{(N-n) \theta^{2}+N \gamma^{2}}{\theta^{2}+\gamma^{2}+N \delta^{2}} & =0 \\
-\frac{\gamma^{2}}{2\left(\theta^{2}+\gamma^{2}\right)}+\frac{\delta^{2}}{\theta^{2}+\gamma^{2}} \frac{(N-n) \theta^{2}+N \gamma^{2}}{\theta^{2}+\gamma^{2}+N \delta^{2}} & =0 .
\end{aligned}
$$

The last expression is decreasing in $n$, which means the second-order condition is satisfied.

Solving for $n$ yields

$$
n^{*}=N\left(1-\frac{\gamma^{2}\left(\theta^{2}+\gamma^{2}-N \delta^{2}\right)}{2 N \delta^{2} \theta^{2}}\right) .
$$

This solution is valid if and only if

$$
0<\gamma^{2}\left(\theta^{2}+\gamma^{2}-N \delta^{2}\right)<2 N \delta^{2} \theta^{2}
$$

i.e. if and only if

$$
\theta^{2}+\gamma^{2}>N \delta^{2}>\gamma^{2}-\frac{\theta^{2} \gamma^{2}}{2 \theta^{2}+\gamma^{2}} .
$$

If $N \delta^{2}>\theta^{2}+\gamma^{2}$ then $n^{*}=N$ (pure $E$-mode is optimal) and if $N \delta^{2}<\gamma^{2}-\frac{\theta^{2} \gamma^{2}}{2 \theta^{2}+\gamma^{2}}$ then $n^{*}=0$ (pure $P$-mode is optimal). Note that $\gamma^{2}-\frac{\theta^{2} \gamma^{2}}{2 \theta^{2}+\gamma^{2}}$ is increasing in $\gamma^{2}$.

\section{Robustness of Corollary 1 to private benefits and spillovers}

Corollary 1 in Section 4.3 of the main paper implies that if $t^{*}<1 / 2$ then the $P$-mode is optimal and if $t^{*}>1 / 2$ then the $E$-mode is optimal. We wish to investigate the extent to which this prediction still holds for the linear demand example once we add private benefits or spillovers.

\subsection{Private benefits}

Using the linear demand example, for any set of parameter values, we can calculate the optimal choice of model and the $t^{*}$ corresponding to the optimal mode. The following parameters need to be specified: $\theta, \delta, \gamma, y$ and $Y$. We normalize $\theta=1$ throughout. We proceed in three steps:

- For a given value of $\delta^{2}$, set $y=Y=0$ and build the set of values of $\gamma^{2}$ such that $t^{E *}$ varies from 0.01 to 0.99 in increments of 0.01 . 
- For each value of $\gamma^{2}$ in the set defined in the previous step, consider 100 equally spaced values of $y$ from 0 to $\gamma^{2}$ and 100 equally spaced values of $Y$ from 0 to $\delta^{2}$. This range of $y$ and $Y$ ensures that $\left(t^{E *}, t^{P *}\right) \in[0,1]^{2}$.

- Repeat the previous two steps for a range of different $\delta^{2}$ as reported in Table 1 below. For each value of $\delta^{2}$, record the average values of $t^{E *}$ and $t^{P *}$ across all 990,000 observations of $\left(\gamma^{2}, y, Y\right)$ defined in the previous steps and the fraction of these observations for which the prediction of Corollary 1 is true.

Table 1

\begin{tabular}{|l|l|l|l|l|l|l|}
\hline$\delta^{2}$ & 0.5 & 1 & 2 & 5 & 10 & 100 \\
\hline$t^{P *}$ & 0.066 & 0.094 & 0.123 & 0.157 & 0.177 & 0.205 \\
$t^{E *}$ & 0.860 & 0.800 & 0.751 & 0.716 & 0.704 & 0.690 \\
\% of obs. theory true & $100 \%$ & $98.9 \%$ & $96.0 \%$ & $91.2 \%$ & $88.5 \%$ & $85.2 \%$ \\
\hline
\end{tabular}

The higher the percentage of observations for which the prediction of Corollary 1 is true, the more confident we can be in using the observed commissions received by agents (or variable fees charged to agents) to infer which mode the firm operates in. Specifically, if we observe agents keeping more than $50 \%$ of variable revenues, then the firm is most likely to be using $P$-mode; if we observe agents keep less than $50 \%$ of variable revenues, then the firm is most likely using the $E$-mode. The values in Table 1 make it clear that this inference is remarkably accurate for low values of $\delta^{2}$.

\section{$7.2 \quad$ Spillovers}

With spillovers rather than private benefits, we have the additional parameter $x$ but no longer have the parameters $y$ and $Y$. Also the parameter $\delta^{2}$ becomes $N \delta^{2}$. The procedure is similar to the case with private benefits.

We normalize $\theta=1$ throughout. We proceed in two steps:

- For a given value of $N \delta^{2}$, build the set of values of $\gamma^{2}$ such that $t^{E *}$ varies from 0.01 to 0.99 in 0.01 intervals (recall that $t^{E *}$ does not depend on $x$ ), and the set of values of $x$ from -0.99 to 0.33 in 0.01 intervals (this is the range of spillovers such that the cross-effect of the transferable action is less than half as important in magnitude as the own effect of the transferable action). ${ }^{3}$

\footnotetext{
${ }^{3}$ Given that the own effect is $\theta-x$, the cross-effect is $x$ and $\theta=1$, this condition requires $x<\frac{1}{2}(1-x)$ for $x>0$ and $|x|<\frac{1}{2}(1+|x|)$ for $x<0$, which is equivalent to $-1<x<\frac{1}{3}$.
} 
- Repeat the previous step for a range of different $N \delta^{2}$ as reported in Table 2 below. For each value of $N \delta^{2}$, record the average values of $t^{E *}$ and $t^{P *}$ across all 13,167 observations of $\left(\gamma^{2}, x\right)$ defined in the previous step and the fraction of these observations for which the prediction of Corollary 1 is true.

Table 2

\begin{tabular}{|l|l|l|l|l|l|l|}
\hline$N \delta^{2}$ & 0.5 & 1 & 2 & 5 & 10 & 100 \\
\hline$t^{P *}$ & 0.213 & 0.238 & 0.257 & 0.273 & 0.280 & 0.286 \\
$t^{E *}$ & 0.883 & 0.848 & 0.818 & 0.793 & 0.782 & 0.771 \\
\% of obs. theory true & $99.2 \%$ & $98.2 \%$ & $96.6 \%$ & $94.3 \%$ & $93.1 \%$ & $92.1 \%$ \\
\hline
\end{tabular}

The results in Table 2 are qualitatively the same as those in Table 1. 